J. Lake Sci. (湖泊科学), 2019, 31(4): 1012-1022

DOI 10. 18307/2019. 0405

(C) 2019 by Journal of Lake Sciences

\title{
三峡水库主要支流碱性磷酸酶活性的比较
}

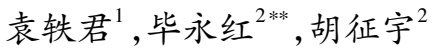 \\ (1: 东华理工大学化学生物与材料科学学院, 南昌 330013) \\ (2: 中国科学院水生生物研究所, 武汉 430072)
}

\begin{abstract}
摘 要: 为进一步认识和理解三峡水库中磷的迁移、转化规律及其与富营养化之间的关系, 于 2014 年 7 月至 2015 年 7 月对三峡水库 12 条典型支流的水体碱性磷酸酶活性进行跟踪观测与比较分析, 同步监测各样点水体的理化指标. 结果 发现, 各支流水体碱性磷酸酶活性的分布呈现显著的时空异质性, 该异质性与各支流水体富营养化程度密切相关. 从季 节上看, 春季碱性磷酸酶活性呈逐渐上升的趋势, 到夏季达到高峰, 秋季碱性磷酸酶活性逐渐下降, 冬季降到全年最低 点. 从空间分布上看, 水体碱性磷酸酶活性在各支流的总体分布趋势为: 小江 $>$ 香溪河 $>$ 苦溪河 $>$ 童庄河 $>$ 青干河 $>$ 袁水河 $>$ 磨刀溪 $>$ 梅溪河 $>$ 汤溪河 $>$ 草堂河 $>$ 大宁河 $>$ 神农溪. 综合营养指数较高的小江、香溪河和茾溪河, 其碱性磷酸酶活性较高; 综合营养指数较低的大宁河和神农溪, 碱性磷酸酶活性较低. 相关分析显示, 各支流碱性磷酸酶活性与水温、溶解氧、电 导率、 $\mathrm{pH}$ 和浊度均存在显著正相关; 与透明度呈显著负相关. 在营养盐方面, 碱性磷酸酶活性与可溶性活性磷呈显著负 相关; 与高锰酸盐指数、叶绿素 a 浓度和综合营养状态指数均呈显著正相关. 不同时期不同生物的贡献和不同环境因素的 影响是导致碱性磷酸酶时空异质性的主要原因.
\end{abstract}

关键词: 碱性磷酸酶; 富营养化; 综合营养状态指数; 三峡水库支流

\section{Comparative study on alkaline phosphatase activity of main tributaries of Three Gorges Reservoir}

\author{
YUAN Yijun $^{1}$, BI Yonghong ${ }^{2 * *} \&$ HU Zhengyu ${ }^{2}$ \\ ( 1: School of Chemistry, Biology and Material Science, East China University of Technology, Nanchang 330013, P.R. China) \\ (2: Institute of Hydrobiology, Chinese Academy of Sciences, Wuhan 430072, P.R.China)
}

\begin{abstract}
To further understand the migration and transformation characteristics of phosphorus and its relationship with eutrophication in the Three Gorges Reservoir, the physical and chemical parameters, alkaline phosphatase activity of 12 typical tributaries of the reservoir were analyzed from July 2014 to July 2015. The research found significant seasonal and regional variations of alkaline phosphatase activity in the tributaries, and the heterogeneity was closely related to the degree of eutrophication. In temporal view, alkaline phosphatase activity was gradually increased from spring to summit in summer. The activity of alkaline phosphatase then dropped from autumn to bottom in winter. On the spatial side, the activity of alkaline phosphatase had high values in Xiaojiang River, Xiangxi River and Zhuxi River, which were at high level of eutrophication. The activity of alkaline phosphatase had low values in Daning River and Shennong River, which were at low level of eutrophication. The correlation analysis results showed that there was a significant positive correlation between alkaline phosphatase activity and water temperature, dissolved oxygen, conductivity, $\mathrm{pH}$ and turbidity; there was a significant negative correlation between alkaline phosphatase activity and transparency in each tributary. In terms of nutrient, alkaline phosphatase activity was significantly negatively correlated with soluble active phosphorus and positive correlated with chemical oxygen demand, chlorophyll-a and trophic state index. The contributions of different organisms and the influences of different environmental factors are the main causes of the spatial and temporal heterogeneity of alkaline phospha-
\end{abstract} tase.

* 江西省教育厅科学技术研究项目 (GJJ160586)、国家自然科学基金项目(31123001) 和中国长江三峡集团公司科研 项目 (CT-12-08-01) 联合资助. 2018-09-30 收稿; 2018-11-28 收修改稿. 袁轶君 (1989 ），男，博士，讲师; E-mail: yuanyijun1229@163.com.

** 通信作者; E-mail:biyh@ihb.ac.cn. 
Keywords: Alkaline phosphatase; eutrophication; trophic state index; tributaries of Three Gorges Reservoir

磷, 作为浮游植物生长的限制性营养元素 ${ }^{[1]}$, 在浮游植物生长过程中起着重要的调控作用. 一方面, 磷 营养浓度的改变会导致藻类群落结构的改变; 另一方面, 藻类能直接利用的磷形态以溶解态反应磷 (SRP) 为主 ${ }^{[2]}$. 因此,水体中的磷代谢尤其依赖于溶解态的有机磷快速转变为 SRP 的循环过程. 碱性磷酸酶可以 催化悬浮态颗粒磷和溶解态有机磷 (DOP) 水解成藻类可直接利用的 SRP, 并在浮游植物吸收利用 DOP 的 过程中起重要作用.

大量的研究表明, 碱性磷酸酶作为一种诱导酶, 其在磷的生物地球化学循环过程中起到了至关重要的 作用 ${ }^{[3-4]}$. 碱性磷酸酶与 SRP 间为典型的“抑制-诱导” 关系. 当水体 SRP 浓度较低时, 藻类、浮游动物、细菌 等会诱导产生大量碱性磷酸酶, 从而将水体中的 DOP 及悬浮态颗粒磷转化成能够被细菌和藻类直接利用的 $\mathrm{SRP}$; 当水体 SRP 充足时, 碱性磷酸酶活性受到抑制 ${ }^{[5-6]}$. 基于此, 许多研究者认为水体生物诱导产生的碱性 磷酸酶是水体生物可利用磷来源的主要补偿机制之一 ${ }^{[7-8]}$, 因此提出将碱性磷酸酶活性作为评价水质的新 生化参数. 碱性磷酸酶与 SRP 间典型的 “抑制-诱导” 关系使得碱性磷酸酶活性可以作为浮游植物磷胁迫水 平的指标 ${ }^{[9-11]}$, 故胞外碱性磷酸酶的活性与水体富营养化程度密切相关.

近些年,磷的地化循环与碱性磷酸酶间的关系广受关注. 然而, 关于碱性磷酸酶的研究工作多集中在海 洋 $^{[9,12]}$ 及浅水湖泊中开展, 如太湖 ${ }^{[7,13]}$ 、西湖 ${ }^{[14]}$ 、东湖 ${ }^{[15]}$ 、白洋淀 ${ }^{[16]}$ 等. 三峡水库中碱性磷酸酶的相关报道 却不多见 ${ }^{[17-19]}$, 有关三峡水库各支流碱性磷酸酶活性及其与水体综合营养状态之间的比较研究更是鲜见报 道. 本研究调查了三峡水库不同营养类型的 12 条支流, 旨在通过对这些支流的连续监测调查, 全面了解各 支流碱性磷酸酶的时空分布差异及其与磷营养的关系, 从而为深人研究三峡水库富营养化演变趋势、碱性 磷酸酶活性与富营养化的相互影响等提供一些理论依据.

\section{1 材料与方法}

\section{1 支流采样点设置}

本研究选取了三峡水库主要的 12 条支流作为研究对象,其中湖北库区的 5 条, 分别为: 神农溪 (SNX,4 个采样点)、青干河 ( 5 个采样点)、袁水河 (3 个采样点)、童庄河 (5 个采样点) 和香溪河 ( 8 个采样点); 重庆 库区的 7 条, 分别为: 苔溪河 ( 1 个采样点)、小江 ( 9 个采样点) 、汤溪河 ( 3 个采样点)、磨刀溪 ( 2 个采样点)、 梅溪河 ( 2 个采样点)、草堂河 (1 个采样点) 和大宁河 ( 3 个采样点) (图 1).

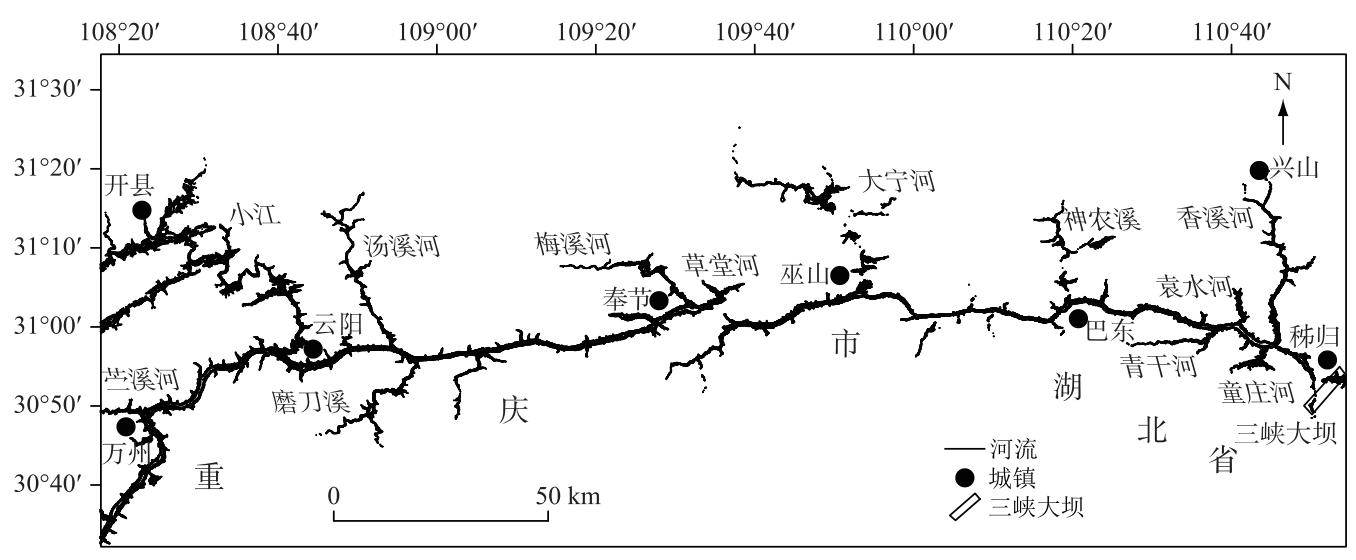

图 1 三峡水库 12 条支流分布

Fig. 1 The location of the 12 tributaries in Three Gorges Reservoir

\section{2 样品采集与分析}

自 2014 年 7 月至 2015 年 7 月,每月采样 1 次. 采样时,利用 Eureka Manta 2 多参数仪(美国) 现场测定 
水温 (WT) 、电导率 ( Cond) 、 $\mathrm{pH}$ 、浊度 ( Turb) 和溶解氧 ( DO), 透明度 $(\mathrm{SD})$ 用赛氏盘法现场测定. 水体中溶解 态反应磷 $(\mathrm{SRP})$ 、总磷 $(\mathrm{TP})$ 、总氮 $(\mathrm{TN})$ 、高锰酸盐指数 $\left(\mathrm{COD}_{\mathrm{Mn}}\right)$ 和叶绿素 $\mathrm{a}(\mathrm{Chl} . \mathrm{a})$ 浓度的测定方法参照《水 和废水监测分析方法》 $\rangle^{[20]}$. 浮游植物定量: 用 $5 \mathrm{~L}$ 采水器取水样 $1.2 \mathrm{~L}$, 用鲁哥试剂现场固定并沉淀浓缩至 $30 \mathrm{ml}$, 在 Olympus BX41 (日本) 显微镜下用浮游植物计数框 $(0.1 \mathrm{ml})$ 计数 ${ }^{[21]}$. 藻类种类鉴定参照文献 [22].

\section{3 碱性磷酸酶活性的测定}

水样中碱性磷酸酶活性采用对硝基苯磷酸二钠比色法测定 ${ }^{[23-25]} .2 \mathrm{ml}$ 水样中加人 Tris- $\mathrm{HCl}$ 缓冲液 $(\mathrm{pH}=8.5$, 浓度 $0.05 \mathrm{~mol} / \mathrm{L}) 1 \mathrm{ml}$, 底物对硝基苯磷酸二钠盐 ( $\mathrm{pNPP}$, 浓度 $0.3 \mathrm{mmol} / \mathrm{L}$ ) $2 \mathrm{ml}$, 置于 $37^{\circ} \mathrm{C}$ 恒温培 养箱反应 $4 \mathrm{~h}$ 后, 加人 $0.1 \mathrm{ml} 0.1 \mathrm{~mol} / \mathrm{L} \mathrm{NaOH}$ 溶液后震荡摇匀, 于 $410 \mathrm{~nm}$ 处利用分光光度法测定吸光值. 用 未经过滤的原水测定水体的碱性磷酸酶总活性 $\left(\mathrm{APA}_{\mathrm{T}}\right)$, 用通过 $3.0 \mu \mathrm{m}$ 孔径滤膜过滤的滤液测定 $\mathrm{APA}_{<3.0 \mu \mathrm{m}}$, 用通过 $0.45 \mu \mathrm{m}$ 孔径滤膜过滤的滤液测定溶解态 $\mathrm{APA}_{<0.45 \mu \mathrm{m}}$. 按下述公式: $\mathrm{APA}_{>3.0 \mu \mathrm{m}}=\mathrm{APA}-$ $\mathrm{APA}_{<3.0 \mu \mathrm{m}}, \mathrm{APA}_{0.45-3 \mu \mathrm{m}}=\mathrm{APA}_{<3.0 \mu \mathrm{m}}-\mathrm{APA}_{<0.45 \mu \mathrm{m}}$, 来计算表征藻类碱性磷酸酶活性的 $\mathrm{APA}_{>3.0 \mu \mathrm{m}}$ 和表征细菌等 微生物的碱性磷酸酶活性 $\mathrm{APA}_{0.45-3 \mu \mathrm{m}}{ }^{[26]}$.

\section{4 数据分析}

数据统计分析运用 SPSS 13.0 软件完成. 碱性磷酸酶活性及理化指标时空差异的分析采用单因素方差 方法进行分析. APA 和理化指标的相关分析采用非参数相关分析的方法. 选用中国环境监测总站推荐的 “湖泊 (水库) 富营养化评价方法及分级技术规定” 中的综合营养状态指数 ${ }^{[27]}$, 以 Chl.a、TP、TN、SD 和 $\mathrm{COD}_{\mathrm{Mn}}$ 为评价指标, 对水体富营养化状况进行评价.

\section{2 结果与分析}

\section{1 水体理化指标的季节变化}

2014 年 7 月至 2015 年 7 月三峡水库 12 条支流的部分理化指标的季节性变化可以看出,各支流的水温 波动在 $12.0 \sim 31.6^{\circ} \mathrm{C}$ 之间, 且具有显著的同步性, 都表现为夏季高冬季低, 各支流之间没有显著性差异 $(P>$ 0.05 ) (图 2). $\mathrm{pH}$ 在 6.9 9.7 之间变化, 其变化趋势与水温相似, 同样表现为夏季高冬季低. 溶解氧、电导率、 浊度和 $\mathrm{COD}_{\mathrm{Mn}}$ 在研究期间不存在显著的季节性差异, 基本保持稳定; 但 12 条支流中莣溪河的溶解氧浓度相 对较低 $(P<0.05)$, 其电导率显著高于其他支流 $(P<0.05)$; 浊度在整个研究期间基本保持恒定, 但是在 2015 年 6 月的小江, 其值达到最高 (177.5 NTU), 原因是期间发生了以微囊藻为优势种的蓝藻水华; 亖溪河的 $\mathrm{COD}_{\mathrm{Mn}}$ 也显著高于其他支流 $(P<0.05)$. 水体透明度变化与水温变化趋势刚好相反, 其值表现为夏季低冬季 高, 且苦溪河的透明度显著低于其他支流 $(P<0.05)$.

在营养盐方面, 同样表现为芒溪河的营养盐浓度显著高于其他支流 $(P<0.05)$. TN 浓度最大值出现在拦 溪河, 为 $4.52 \pm 1.51 \mathrm{mg} / \mathrm{L}$, 而其他支流的 TN 平均浓度都在 $2 \mathrm{mg} / \mathrm{L}$ 以下. 贲溪河的 TP 平均浓度也最高, 为 $0.31 \pm 0.14 \mathrm{mg} / \mathrm{L}$, 香溪河次之, 为 $0.16 \pm 0.05 \mathrm{mg} / \mathrm{L}, 2015$ 年 6 月小江 TP 浓度出现峰值可以解释小江蓝藻水华 的发生. SRP 浓度仍然是贲溪河最高, 为 $0.15 \pm 0.1 \mathrm{mg} / \mathrm{L}$, 香溪河次之, 为 $0.09 \pm 0.03 \mathrm{mg} / \mathrm{L}($ 表 1$)$.

叶绿素 a 浓度在研究期间也存在显著的季节性差异,其表现为春、夏季较高, 而秋、冬季较低. 亖溪河平 均叶绿素 a 浓度最高, 为 $83.43 \pm 82.17 \mu \mathrm{g} / \mathrm{L}$, 显著高于其他支流 $(P<0.05)$; 大宁河叶绿素 a 浓度最低, 年均值 小于 $10 \mu \mathrm{g} / \mathrm{L}$.

\section{2 水体富营养化状况分析}

研究期间, 各支流水体的综合营养状况 (表现为春、夏季水体 TLI 较高, 大多处于轻度富营养状态; 而 秋、冬季相对较低, 除贲溪河外所有的支流都处于中营养状态 (图 3). 苦溪河全年的 $T L I$ 值都高于其他支流, 在春夏季甚至处于重度富营养状态. 从 $T L I$ 的年平均值来看 (表 2), 亖溪河的富营养化程度显著高于其他支 流 $(P<0.05)$, 其值为 $70.56 \pm 14.34$, 处于重度富营养化状态; 大宁河、神农溪 $T L I$ 值分别为 $48.71 \pm 8.01 、 49.94 \pm$ 8.48 , 处于中营养状态; 而其他支流水体都处于轻度富营养化状态. 

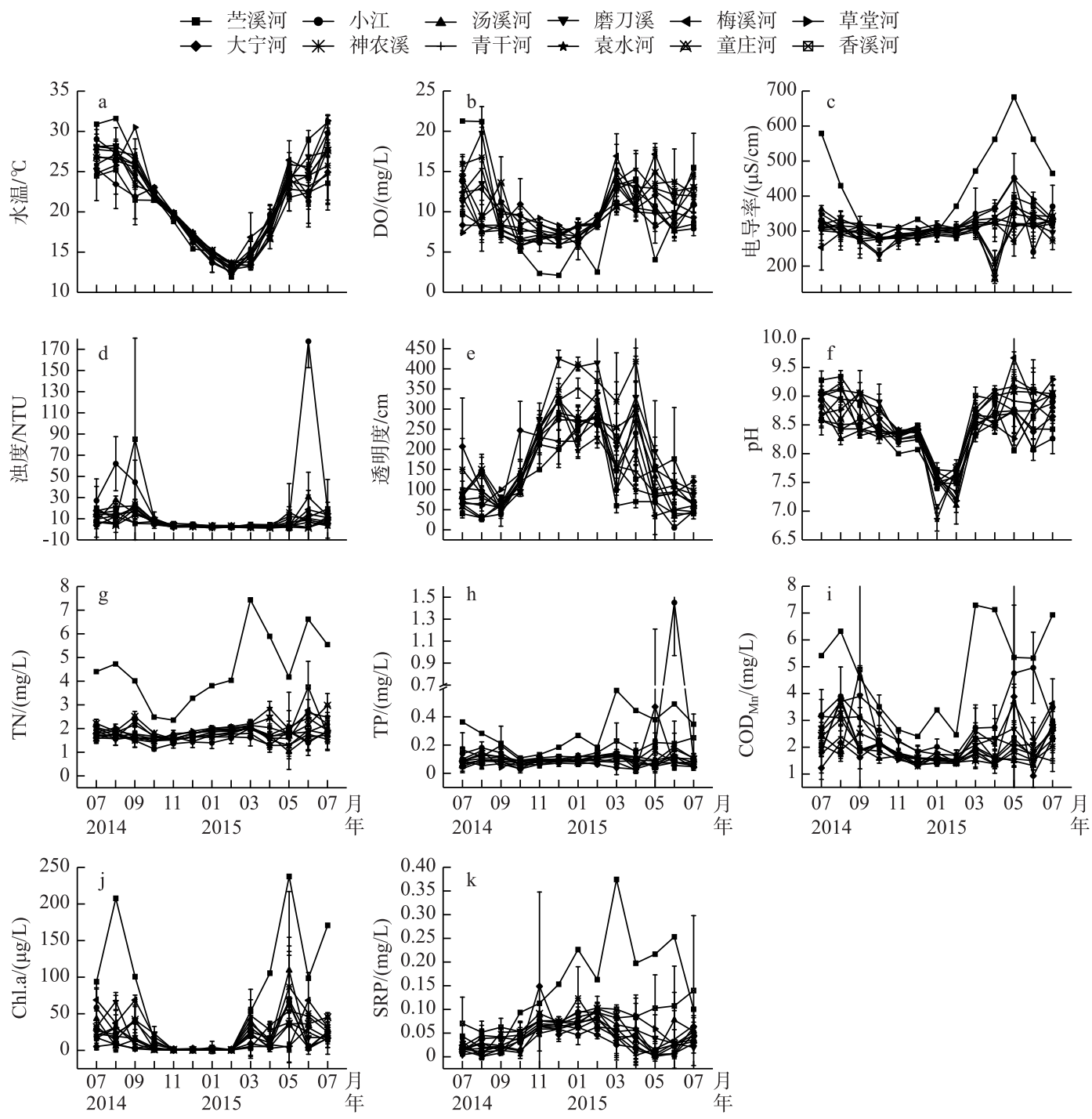

图 22014 年 7 月至 2015 年 7 月三峡水库 12 条支流理化因子的周年性季节变化

Fig.2 Seasonal changes of physico-chemical characteristics of the 12 tributaries in Three Gorges Reservoir during July 2014 to July 2015

\section{3 碱性磷酸酶活性季节变化}

在整个研究过程中, 碱性磷酸酶活性在 $5.6 \sim 114.8 \mathrm{nmol} / \mathrm{L} \cdot \min )$ 范围内波动, 最大值出现在 2015 年 6 月的小江, 其原因是期间暴发了以微囊藻为优势种的蓝藻水华. 碱性磷酸酶活性存在显著的季节性差异, 夏 季 (6-8 月) 水体中碱性磷酸酶平均活性最高, 为 $17.8 \mathrm{nmol} /(\mathrm{L} \cdot \mathrm{min})$; 秋季 $(9-11$ 月 $)$ 次之, 平均碱性磷酸 酶活性为 $11.6 \mathrm{nmol} / \mathrm{L} \cdot \mathrm{min})$; 冬季 $(12-2$ 月) 和春季 $(3-5$ 月)平均碱性磷酸酶活性相对较低, 分别为 7.3 和 $10.4 \mathrm{nmol} /(\mathrm{L} \cdot \mathrm{min})$. 在三峡水库高水位时期的秋冬季, 各支流之间的碱性磷酸酶活性不存在显著性差异 (图 4). 
表 1 三峡水库 12 条支流叶绿素 a 浓度、可溶性活性磷与环境因子的年度均值

Tab.1 The annual means of Chl.a,SRP and environmental variables of 12 tributaries in Three Gorges Reservoir

\begin{tabular}{|c|c|c|c|c|c|c|c|c|c|c|c|}
\hline 支流 & $\begin{array}{c}\text { 水温/ } \\
{ }^{\circ} \mathrm{C}\end{array}$ & $\begin{array}{c}\text { 溶解氧/ } \\
(\mathrm{mg} / \mathrm{L})\end{array}$ & $\begin{array}{l}\text { 电导率/ } \\
(\mu \mathrm{S} / \mathrm{cm})\end{array}$ & $\begin{array}{l}\text { 浊度/ } \\
\text { NTU }\end{array}$ & $\begin{array}{c}\text { 透明度/ } \\
\mathrm{cm}\end{array}$ & $\mathrm{pH}$ & $\begin{array}{c}\mathrm{TN} / \\
(\mathrm{mg} / \mathrm{L})\end{array}$ & $\begin{array}{c}\mathrm{TP} / \\
(\mathrm{mg} / \mathrm{L})\end{array}$ & $\begin{array}{l}\mathrm{COD}_{\mathrm{Mn}} / \\
(\mathrm{mg} / \mathrm{L})\end{array}$ & $\begin{array}{c}\text { 叶绿素 a/ } \\
(\mu \mathrm{g} / \mathrm{L})\end{array}$ & $\begin{array}{c}\mathrm{SRP} / \\
(\mathrm{mg} / \mathrm{L})\end{array}$ \\
\hline 亖溪河 & $22.3 \pm 7.0$ & $9.9 \pm 6.8$ & $438.4 \pm 126.8$ & $7.5 \pm 4.7$ & $110 \pm 89$ & $8.4 \pm 0.6$ & $4.5 \pm 1.5$ & $0.31 \pm 0.14$ & $4.8 \pm 1.8$ & $83.4 \pm 82.20$ & $0.15 \pm 0.10$ \\
\hline 小江 & $21.3 \pm 5.8$ & $8.9 \pm 3.1$ & $313.4 \pm 59.5$ & $28.3 \pm 48.5$ & $132 \pm 97$ & $8.3 \pm 0.4$ & $1.6 \pm 0.3$ & $0.21 \pm 0.37$ & $3.0 \pm 1.0$ & $21.0 \pm 22.60$ & $0.04 \pm 0.02$ \\
\hline 汤溪河 & $20.8 \pm 5.4$ & $9.1 \pm 2.3$ & $320.1 \pm 18.8$ & $9.2 \pm 9.7$ & $157 \pm 108$ & $8.3 \pm 0.5$ & $1.7 \pm 0.3$ & $0.11 \pm 0.03$ & $1.9 \pm 0.6$ & $18.0 \pm 30.20$ & $0.06 \pm 0.03$ \\
\hline 磨刀溪 & $21.5 \pm 5.8$ & $9.7 \pm 1.8$ & $301.6 \pm 34.8$ & $4.8 \pm 4.8$ & $221 \pm 134$ & $8.5 \pm 0.6$ & $1.7 \pm 0.2$ & $0.07 \pm 0.02$ & $2.5 \pm 0.8$ & $19.0 \pm 19.70$ & $0.04 \pm 0.03$ \\
\hline 梅溪河 & $22.0 \pm 5.6$ & $11.1 \pm 3.5$ & $299.4 \pm 26.3$ & $8.2 \pm 6.2$ & $132 \pm 91$ & $8.6 \pm 0.6$ & $1.8 \pm 0.3$ & $0.11 \pm 0.03$ & $2.4 \pm 0.8$ & $26.9 \pm 27.80$ & $0.04 \pm 0.02$ \\
\hline 草堂河 & $21.8 \pm 6.0$ & $9.7 \pm 2.2$ & $336.9 \pm 43.7$ & $6.8 \pm 5.4$ & $168 \pm 107$ & $8.5 \pm 0.6$ & $1.9 \pm 0.2$ & $0.10 \pm 0.03$ & $1.9 \pm 0.7$ & 11.2 & \pm 0.03 \\
\hline 大宁河 & $20.1 \pm 4.3$ & $9.6 \pm 1.7$ & $306.7 \pm 25.1$ & $5.9 \pm 5.2$ & $190 \pm 87$ & 0.4 & $1.6 \pm 0.2$ & $0.09 \pm 0.12$ & $1.8 \pm 0.7$ & $8.4 \pm 10.3$ & $0.04 \pm 0.04$ \\
\hline 神农溪 & $21.0 \pm 5.2$ & $10.8 \pm 3.4$ & $301.7 \pm 19.7$ & $6.4 \pm 6.7$ & $223 \pm 134$ & $8.6 \pm 0.6$ & $1.6 \pm 0.2$ & $0.08 \pm 0.03$ & $1.8 \pm 0.6$ & 18.8 & $0.04 \pm 0.03$ \\
\hline 青干河 & $20.6 \pm 4.6$ & $10.3 \pm 3.3$ & $295.8 \pm 26.7$ & $6.2 \pm 5.6$ & $167 \pm 105$ & $8.5 \pm 0.6$ & $1.8 \pm 0.5$ & $0.08 \pm 0.03$ & $1.8 \pm 0.3$ & $16.6 \pm 16.3$ & $0.04 \pm 0.02$ \\
\hline 袁水河 & $21.4 \pm 5.3$ & $10.9 \pm 4.1$ & $297.8 \pm 31.3$ & $8.0 \pm 7.3$ & $141 \pm 84$ & $8.6 \pm 0.7$ & $1.9 \pm 0.6$ & $0.10 \pm 0.02$ & $2.2 \pm 0.5$ & $20.7 \pm 23.8$ & $0.04 \pm 0.03$ \\
\hline 童庄河 & $21.0 \pm 5.2$ & $10.5 \pm 3.1$ & $304.8 \pm 49.4$ & $5.5 \pm 5.0$ & $175 \pm 93$ & $8.6 \pm 0.5$ & $1.9 \pm 0.6$ & $0.09 \pm 0.02$ & $1.9 \pm 0.4$ & $19.4 \pm 17.9$ & $0.05 \pm 0.03$ \\
\hline 香溪河 & $19.6 \pm 4.1$ & $9.0 \pm 2.2$ & $300.0 \pm 51.7$ & $13.3 \pm 22.5$ & $167 \pm 83$ & $8.4 \pm 0.4$ & $1.8 \pm 0.8$ & $0.16 \pm 0.05$ & $2.0 \pm 0.9$ & $12.3 \pm 17.2$ & $0.09 \pm 0.03$ \\
\hline
\end{tabular}

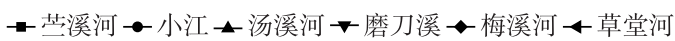

$\rightarrow$ 大宁河 $*$ 神农溪 - 青干河 $*$ 袁水河 $\prec$ 童庄河 一香溪河

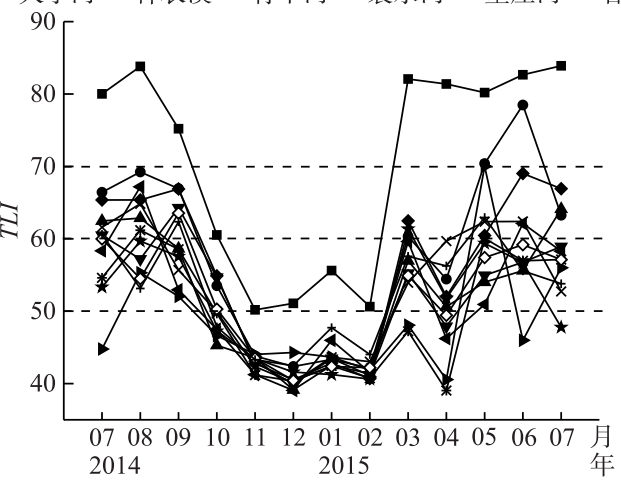

图 32014 年 7 月至 2015 年 7 月 三峡水库 12 条支流 $T L I$ 的时空变化

Fig. 3 Spatio-temporal dynamics of $T L I$ of the 12 tributaries in Three Gorges Reservoir during July 2014 to July 2015

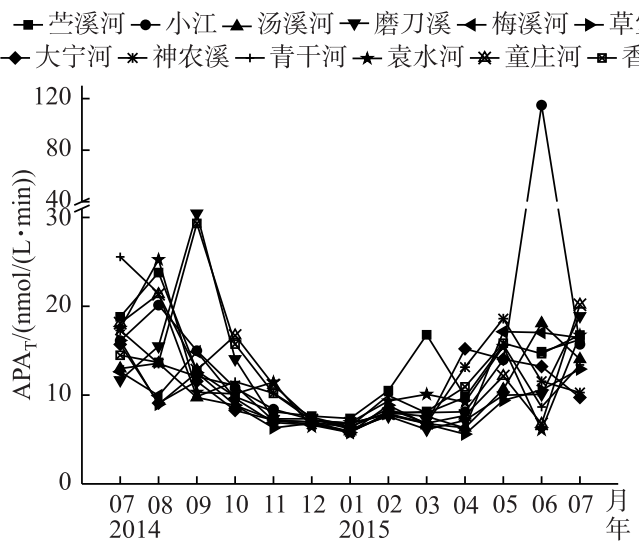

图 42014 年 7 月至 2015 年 7 月 三峡水库 12 条支流 $\mathrm{APA}_{\mathrm{T}}$ 的季节变化

Fig.4 Seasonal changes of $\mathrm{APA}_{\mathrm{T}}$ of the 12 tributaries in Three Gorges Reservoir during July 2014 to July 2015

\section{4 碱性磷酸酶活性大小分级}

图 5 展示了研究期间 12 条主要支流的碱性磷酸酶的分级组成情况. 从空间上看, 各支流碱性磷酸酶组 成差异不显著, 以溶解态 $\left(\mathrm{APA}_{<0.45 \mu \mathrm{m}}\right)$ 为主, 约占碱性磷酸酶活性的 $67.7 \%$; 其次是大颗粒的碱性磷酸酶 $\left(\mathrm{APA}_{>3.0 \mu \mathrm{m}}\right)$, 占碱性磷酸酶活性的 $22.3 \%$; 而细菌的碱性磷酸酶 $\left(\mathrm{APA}_{0.45-3 \mu \mathrm{m}}\right)$ 活性较低, 占碱性磷酸酶活性 的 $11 \%$. 在碱性磷酸酶活性较高的茾溪河和香溪河, 大颗粒碱性磷酸酶所占比例也较高, 分别为 $24 \%$ 和 $31 \%$; 而在碱性磷酸酶活性较低的汤溪河、梅溪河和草堂河, 大颗粒碱性磷酸酶所占比例较低,分别为 $16 \%$ 、 $19 \%$ 和 $15 \%$. 从季节上看, 碱性磷酸酶组成存在季节性差异, 在综合营养状态指数较高的夏季, $\mathrm{APA}_{>3.0 \mu \mathrm{m}}$ 所 占的比例要显著高于冬季. 在小江暴发蓝藻水华的 2015 年 6 月, $\mathrm{APA}_{>3.0 \mu \mathrm{m}}$ 占碱性磷酸酶的比例甚至高 达 $88 \%$.

在 12 条支流中, 亖溪河、小江和香溪河的碱性磷酸酶活性相对较高, 其全年平均值分别为 $13.0 、 19.4$ 和 
$13.2 \mathrm{nmol} /(\mathrm{L} \cdot \min )$; 而汤溪河、梅溪河、草堂河、大宁 河和神农溪的碱性磷酸酶活性相对较低, 其年均值 均低于 $11 \mathrm{nmol} /(\mathrm{L} \cdot \mathrm{min})$, 处于中营养状态的大宁河 和神农溪, 其碱性磷酸酶活性年平均值分别为 10.9 和 $10.8 \mathrm{nmol} /(\mathrm{L} \cdot \mathrm{min})$. 水体碱性磷酸酶活性在各支 流的总体分布趋势为: 小江 $>$ 香溪河 $>$ 苦溪河 $>$ 童庄 河 $>$ 青干河 $>$ 袁水河 $>$ 磨刀溪 $>$ 梅溪河 $>$ 汤溪河 $>$ 草堂 河 $>$ 大宁河 $>$ 神农溪.

\section{5 相关性分析}

通过分析三峡水库 12 条主要支流 $\mathrm{APA}_{\mathrm{T}}$ 、叶绿 素 a 及理化指标等参数间的 Spearman 相关系数发 现, 各支流碱性磷酸酶活性与水温、溶解氧、电导率、 $\mathrm{pH}$ 和浊度均存在显著的正相关关系; 与透明度具有 显著的负相关关系. 叶绿素 a 浓度与水温、溶解氧、 电导率、 $\mathrm{pH}$ 和浊度均存在显著的正相关关系; 与透 明度存在显著的负相关 (表 3 ).

在营养盐方面, 碱性磷酸酶活性与可溶性活性磷呈显著的负相关关系; 与高锰酸盐指数、叶绿素 a 浓度 和 TLI 均存在显著的正相关关系. 叶绿素 a 浓度与可溶性活性磷具有显著的负相关关系; 与总氮浓度和高锰 酸盐指数有显著的正相关关系.

\section{表 3 三峡水库 12 条支流碱性磷酸酶活性、综合营养状态指数、叶绿素 a 与 环境因子的 Spearman 相关系数矩阵 $(n=156)$}

Tab.3 Spearman rank correlation among $\mathrm{APA}_{\mathrm{T}}, T L I$, chlorophyll-a and environmental variables of the 12 tributaries in Three Gorges Reservoir $(n=156)$

\begin{tabular}{|c|c|c|c|c|c|c|c|c|c|c|c|c|c|}
\hline 相关系数 & $\mathrm{APA}_{\mathrm{T}}$ & WT & DO & Cond & $\mathrm{pH}$ & Turb & SD & SRP & $\mathrm{TP}$ & $\mathrm{TN}$ & $\mathrm{COD}_{\mathrm{Mn}}$ & Chl.a & $T L I$ \\
\hline $\mathrm{APA}_{\mathrm{T}}$ & 1.000 & & & & & & & & & & & & \\
\hline WT & $0.722^{* *}$ & 1.000 & & & & & & & & & & & \\
\hline DO & $0.323^{* * *}$ & $0.354^{\text {*** }}$ & * 1.000 & & & & & & & & & & \\
\hline Cond & $0.272^{* * *}$ & $0.262^{\text {*** }}$ & * $0.312^{* *}$ & 1.000 & & & & & & & & & \\
\hline $\mathrm{pH}$ & $0.537^{* *}$ & $0.665^{* * *}$ & * $0.767^{* *}$ & 0.180 & 1.000 & & & & & & & & \\
\hline Turb & $0.576^{\text {*** }}$ & $0.685^{\text {*** }}$ & * 0.04 & $0.088^{*}$ & $0.297^{\text {** }}$ & * 1.000 & & & & & & & \\
\hline SD & $-0.633^{* *}$ & $-0.765^{\text {** }}$ & $* 0.336$ * & $-0.236 * *$ & $*-0.5277^{* *}$ & $* 0.804$ ** & * 1.000 & & & & & & \\
\hline SRP & -0.470 ** & $-0.576^{* *}$ & $* 0.482^{* *}$ & 0.054 & $-0.656^{* *}$ & $*-0.344^{* *}$ & * 0.444 ** & 1.000 & & & & & \\
\hline $\mathrm{TP}$ & -0.009 & -0.054 & -0.032 & 0.329 ** & $*-0.110$ & $0.189^{*}$ & $-0.226^{* *}$ & $0.428^{* * *}$ & * 1.000 & & & & \\
\hline $\mathrm{TN}$ & 0.067 & -0.059 & $0.186^{*}$ & $0.353^{* *}$ & * 0.049 & -0.034 & -0.135 & $0.223^{* *}$ & * $0.478^{* * *}$ & * 1.000 & & & \\
\hline $\mathrm{COD}_{\mathrm{Mn}}$ & $0.472 * *$ & $0.537^{* * *}$ & * 0.308 ** & $0.189^{*}$ & $0.433^{* *}$ & $* 0.499^{* *}$ & $* 0.650 * *$ & $-0.270^{* *}$ & * 0.278 *** & * $0.183^{*}$ & 1.000 & & \\
\hline Chl.a & $0.555^{* * *}$ & $0.622^{* * *}$ & * $0.693^{* *}$ & $0.449^{* *}$ & * 0.774 ** & $* 0.384^{* *}$ & $* 0.665$ ** & $-0.508^{* *}$ & 0.128 & $0.207^{\text {*** }}$ & 0.597 *** & 1.000 & \\
\hline$T L I$ & $0.212^{* *}$ & $0.197^{*}$ & 0.126 & 0.027 & $0.161^{*}$ & 0.114 & -0.087 & -0.143 & -0.035 & -0.040 & 0.036 & 0.115 & 1.000 \\
\hline
\end{tabular}

${ }^{*} P<0.05,{ }^{* *} P<0.01$.

\section{3 讨论}

周易勇等 ${ }^{[28-29]}$ 在对东湖磷酸酶的研究中指出, 碱性磷酸酶能补充湖泊的磷营养, 指示磷的丰缺,介导磷 的循环, 具有生态学意义. 基于此, 弄清楚三峡水库各支流碱性磷酸酶活性的时空分布有助于了解水体磷形 态的转化途径、浮游生物的分布特征以及水华暴发的潜在性. 三峡水库碱性磷酸酶活性的时空分布呈现非 均一性的特征. 从图 2 中可以看出, 三峡水库各支流水体中碱性磷酸酶活性呈现明显的时间异质性. 整体上 

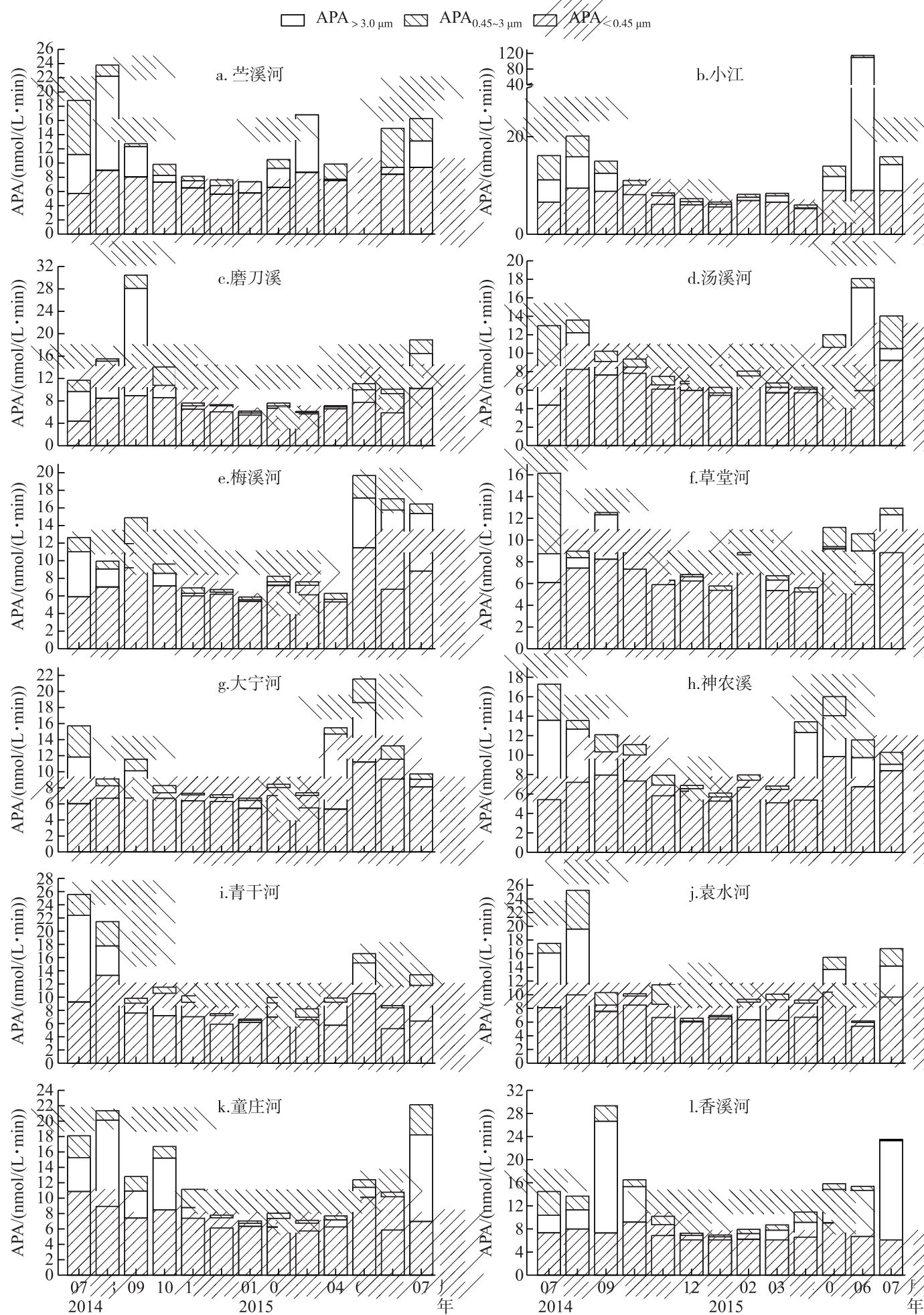

图 5 三峡水库 12 条支流的碱性磷酸酶分级

Fig.5 Average size-fractionated APA of the 12 tributaries in Three Gorges Reservoir 
看, 春季碱性磷酸酶活性呈逐渐上升的趋势, 到夏季达到高峰, 秋季碱性磷酸酶活性逐渐下降, 冬季降到全 年最低点. 通过比较发现, 各支流碱性磷酸酶活性的季节变化趋势和各支流水体的综合营养状态指数的变 化趋势 (图 3) 非常的一致. 各支流水体碱性磷酸酶活性的空间分布也呈现非均一性的特征 (图 5). 水体碱 性磷酸酶活性在各支流的总体分布趋势为: 小江 $>$ 香溪河 $>$ 荁溪河 $>$ 童庄河 $>$ 青干河 $>$ 袁水河 $>$ 磨刀溪 $>$ 梅溪 河 $>$ 大宁河 $>$ 神农溪>汤溪河 $>$ 草堂河. 该趋势与不同支流水体的 $T L I$ 的变化趋势也很相似,在处于重度富营 养化状态的苦溪河, 其 $\mathrm{APA}_{\mathrm{T}}$ 较高; 而在 $T L I$ 值较低的大宁河和神农溪, $\mathrm{APA}_{\mathrm{T}}$ 值较低. 周易勇等 ${ }^{[29]}$ 研究表明, 武汉东湖表层水体中碱性磷酸酶活性也呈现出非均一性分布特征, 这与本研究的结论一致. 章婷曦等 ${ }^{\left[{ }^{[} 0\right]}$ 在 太湖的研究证实, 碱性磷酸酶活性的空间异质性分布与污染程度有关. 污染程度高, 水动力条件差, 水体交 换能力弱的点位碱性磷酸酶活力高. 通过比较研究期间 12 条主要支流的 $\mathrm{APA}_{\mathrm{T}}$, 发现各支流水体碱性磷酸 酶活性与水体的综合营养状况无论是在空间分布还是在季节变化上都具有非常相似的一致性. 这说明水体 碱性磷酸酶活性与富营养化程度密切相关. 在英国 16 个富营养化湖泊中, 水体碱性磷酸酶活力正比于水体 的富营养化程度 ${ }^{[31]}$. 本研究通过对以叶绿素浓度表征的浮游植物生物量、水体综合营养状态指数和水体碱 性磷酸酶活性的相关分析发现, $\mathrm{APA}_{\mathrm{T}}$ 与叶绿素 $\mathrm{a}$ 浓度、TLI 值均表现为显著的正相关关系. 综上所述, 水体 碱性磷酸酶活性与富营养化程度密切相关.

本研究中, 三峡水库各支流水体碱性磷酸酶的时空分布的异质性, 可能源于不同时期不同环境因素的 影响和不同生物的贡献. 碱性磷酸酶活性与环境因子的相关分析表明, 影响酶活性的因素是多方面且复杂 的,包括非生物因子水温、透明度和营养盐以及生物因子浮游植物等等. 温度可以影响酶活性, 低温抑制酶 活性, 一定范围内温度升高则会使酶活性增加. 研究表明, 水体碱性磷酸酶活性表达的最佳温度范围为 25 $40^{\circ} \mathrm{C}$, 温度过高或过低均不利于活性的表达 ${ }^{[28]}$. 表 3 中的相关分析结果表明, 温度与碱性磷酸酶活性呈显著 正相关 $(R=0.722, P<0.01)$. 因此, 在一年当中温度最低的冬季, 所测到的碱性磷酸酶活性也最低; 春季随着 温度上升, 酶活性开始上升; 到了夏季温度达到最高, 酶活性也达到最大; 而随着秋季温度逐渐下降, 碱性磷 酸酶活性也逐渐下降.

除了温度, 营养盐对碱性磷酸酶活性也有显著影响, 营养盐浓度对有机物的分解率及酶活性有促进作

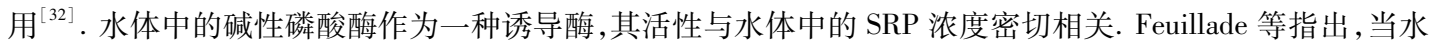
体中 SRP 浓度不足以维持浮游植物、细菌生长时, 藻类、细菌碱性磷酸酶基因受到诱导并表达, 其胞外磷酸 酶活性显著地增加 ${ }^{[33]}$. 因此, 作为碱性磷酸酶的主要载体的浮游植物, 其生物量与碱性磷酸酶活性息息相 关. 表 3 中的分析结果显示, 叶绿素 a 浓度与碱性磷酸酶活性呈显著正相关 $(R=0.555, P<0.01)$. 所以在水 体综合营养指数较高的草溪河和小江, 藻类生物量较大, 因此对碱性磷酸酶的贡献率也较大. 大宁河和神农 溪水体较其他支流富营养化程度较低, 水中碱性磷酸酶活性相对较低. 研究发现富营养化的湖泊与海湾中 碱性磷酸酶活性较高, 与本实验结果一致 ${ }^{[31,34]}$. 而香溪河和童庄河水体中碱性磷酸酶活性却表现出明显不 同的特征, 富营养化程度相对较低的水域酶活性却相对较高, 这可能与这两条支流的自然地理环境和当地 人类活动关系密切. 香溪河为含高磷岩层地质环境, 流域内的磷矿资源丰富, 与磷相关产业已是兴山县的支 柱产业 ${ }^{[35]}$. 童庄河周边的人口密度较大, 常年接纳大量的生活污水; 并且两岸还有较多种植业 ${ }^{[36]}$. 生活污水 和工业废水中的磷随着地表径流积蓄在沉积物中, 在水一沉积物界面碱性磷酸酶的调控下, 会以内源磷释放 的形式重新进人水体. 事实上, 水体中碱性磷酸酶的诱导和抑制是一个极其复杂的过程. 一系列相关的研究 证明, 浮游植物在磷胁迫状态下, 可通过分泌碱性磷酸酶水解有机磷来补充可利用性磷, 本实验所有支流 中, APA 与 SRP 浓度同样也呈现显著的负相关关系. 因此碱性磷酸酶活性可用于指示浮游植物的磷胁迫状 态 ${ }^{[10-11]}$. 而在很多情况下, 碱性磷酸酶的活性不受磷水平的调节. 加拿大湖水水样中大量加人无机磷之后, 磷酸酶的活性并未明显降低 ${ }^{[37]}$. 曹秀云等 ${ }^{[38]}$ 在对梁子湖等浅水湖泊的研究中发现, 由于细菌及浮游植物分 泌碱性磷酸酶的种差异性导致 APA 与 SRP 呈显著正相关. 大量的研究证实 SRP 对碱性磷酸酶活性的调节 作用存在于一定的 SRP 浓度范围内, 且不同的水体具有不同的阈值浓度. 洪华生等 ${ }^{[39]}$ 推测, SRP 对 APA 的 作用主要是在 SRP 较低时发生, 并且在某一界限以上时, SRP 对 APA 的影响是不显著的. Chróst 等 ${ }^{[40]}$ 的实 验表明, 当 $\mathrm{SRP}$ 浓度低于 $0.01 \mathrm{mg} / \mathrm{L}$ 时, $\mathrm{APA}_{\mathrm{T}}$ 明显增加. 而对于太湖来说, $0.02 \mathrm{mg} / \mathrm{L}$ 的 $\mathrm{SRP}$ 浓度可能是激 发浮游植物体中 APA 的阈值浓度; 当水体中的 SRP 浓度大于 $0.02 \mathrm{mg} / \mathrm{L}$ 时, 可能对 APA 产生抑制, 致使其 
活性显著降低 ${ }^{[20]}$. 而通过我们对香溪河的研究发现, $0.09 \mathrm{mg} / \mathrm{L}$ 的 SRP 浓度可能是激发浮游植物中 APA 的 阈值浓度 (待发表). 因此, 在特定的 SRP 临界浓度范围内, APA 可用于评价水体中浮游植物的磷胁迫状态.

\section{4 结论}

三峡水库 12 条支流中碱性磷酸酶的分布呈现一定的时空异质性,且与富营养化之间有着一定的相互 关系, 随着富营养化程度的增加, 酶活性也具有增加的趋势. 从季节上看, 随着春季温度回升, 碱性磷酸酶的 活性得以激发、增大, 到夏季达到高峰, 秋季酶活性逐渐降低, 冬季降到全年最低点; 从空间上看, 富营养化 程度高的小江和荁溪河碱性磷酸酶活性较高, 而在 TLI 值较低的大宁河和神农溪, $\mathrm{APA}_{\mathrm{T}}$ 值较低. 总之, 水体 碱性磷酸酶活性与富营养化程度密切相关, 在特定的 SRP 临界浓度范围内可作为评价浮游植物磷胁迫状态 的指标.

\section{5 参考文献}

[ 1 ] Carpenter SR. Phosphorus control is critical to mitigating eutrophication. Proceedings of the National Academy of Sciences of the United States of America, 2008, 105(32) : 11039-11040. DOI: 10.1073/pnas.0806112105.

[ 2 ] Peterson BJ, Barlow JP, Savage AE. The physiological state with respect to phosphorus of Cayuga Lake phytoplankton. Limnology and Oceanography, 1974, 19(3) : 396-408. DOI: 10.4319/lo.1974.19.3.0396.

[ 3 ] Zhou YY, Song CL, Cao XY et al. Phosphorus fractions and alkaline phosphatase activity in sediments of a large eutrophic Chineses lake (Lake Taihu). Hydrobiologia, 2008, 599(1) : 119-125. DOI: 10.1007/s10750-007-9185-z.

[ 4 ] Zhu YL, Wu FC, Feng WY et al. Interaction of alkaline phosphatase with minerals and sediments : activities, kinetics and hydrolysis of organic phosphorus. Colloids and Surfaces A: Physicochemical and Engineering Aspects, 2016, 495: 46-53. DOI: $10.1016 /$ j.colsurfa.2016.01.056.

[ 5 ] Berman T. Alkaline phosphatases and phosphorus availability in Lake Kinneret. Limnology and Oceanography, 1970,15 : 663-674. DOI: 10.4319/lo.1970.15.5.0663.

[ 6 ] Jansson M, Olsson H, Pettersson K. Phosphatases: origin, characteristics and function in lakes. Hydrobiologia, 1988, 170(1) : 157-175. DOI: 10.1007/BF00024903.

[ 7 ] Gao G, Zhu GW, Qin BQ et al. Activity of alkaline phosphatase and its mineralization rate in Taihu Lake. Science in China: Ser D: Earth Sciences, 2005, 35(S2) :157-165. [高光, 朱广伟, 秦伯强等. 太湖水体中碱性磷酸酶的活性及磷 的矿化速率. 中国科学: D 辑: 地球科学, 2005, 35(S2):157-165.]

[ 8 ] Rose C, Axler RP. Uses of alkaline phosphatase activity in evaluating phytoplankton community phosphorus deficiency. Hydrobiologia, 1997, 361 (1/2/3) : 145-156. DOI: 10.1023/A:1003178502883.

[ 9 ] Huang BQ, Ou LJ, Wang XL et al. Alkaline phosphatase activity of phytoplankton in East China Sea coastal waters with frequent harmful algal bloom occurrences. Aquatic Microbial Ecology, 2007, 49: 195-206. DOI: 10.3354/ame01135.

[10] Liu XH, Qin XL, Cai YY et al. Alkaline phosphatase activity of phytoplankton in Qinhuangdao coastal waters with frequent brown tide occurrences. Marine Sciences, 2016, 40(3) : 95-100. DOI: 10.11759/hykx20150925001. [刘晓红, 覃仙玲, 蔡阳扬等. 秦皇岛近海褐潮高发区浮游植物的碱性磷酸酶活性分析. 海洋科学, 2016, 40(3) : 95-100.]

[11] Qin XL, Chen B, Lai JX et al. Phosphorus nutrition state and alkaline phosphatase activity of phytoplankton in the Qinzhou Bay. Marine Science Bulletin, 2018, 37(2) : 169-176. [覃仙玲, 陈波, 赖俊翔等. 钦州湾磷营养状态与浮游 植物的碱性磷酸酶活性分析. 海洋通报, 2018, 37(2): 169-176.]

[12] Wang D, Huang BQ, Liu X et al. Seasonal variations of phytoplankton phosphorus stress in the Yellow Sea Cold Water Mass. Acta Oceanologica Sinica, 2014, 33(10) : 124-135. DOI: 10.1007/s13131-014-0547-x.

[13] Gao G, Gao XY, Qin BQ et al. Experimental study on the $\mathrm{PO}_{4}^{3-}-\mathrm{P}$ threshold of the alkaline phosphatase activity in Taihu Lake. J Lake Sci, 2000, 12(4) :353-358. DOI: 10.18307/2000.0409. [高光, 高锡云, 秦伯强等. 太湖水体中碱性磷 酸酶的作用阈值. 湖泊科学, $2000,12(4): 353-358$. ]

[14] Chen YY, Zhu YM, Hu ML et al. The relationship between enzymatic activity and nutrition release in the sediment of West Lake. Journal of Zhejiang Agricultural University, 1997, 23(2): 171-174. [陈宜宜, 朱荫湄, 胡木林等. 西湖底泥中 酶活性与养分释放的关系. 浙江农业大学学报, 1997, 23(2):171-174.]

[15] Zhou YY, Li JQ, Zhang M et al. Distribution of kinetic parameters of alkaline phosphatase in sediments of shallow lakes. $J$ 
Lake Sci, 2001, 13(3) :261-266. DOI: 10.18307/2001.0310. [ 周易勇, 李建秋, 张敏等. 浅水湖泊中沉积物碱性磷 酸酶动力学参数的分布. 湖泊科学, $2001, \mathbf{1 3}(3)$ : 261-266.]

[16] Xu S, Liu CQ, Dong MH et al. The spatio-temporal variation of alkaline phosphatase activity in water and sediment of Baiyangdian Lake and its impact factors. Acta Scientiae Circumstantiae, 2013, 33(12):3317-3323. DOI: 10.13671/j. hjkxxb.2013.12.022. [徐爽, 刘存歧, 董梦荟等. 白洋淀水体和沉积物中碱性磷酸酶活性的时空分布及其影响因 素. 环境科学学报, 2013, 33(12):3317-3323.]

[17] Yuan YJ, Bi YH, Zhu KX et al. Alkaline phosphatase activity in the sediments of the Three Gorges Reservoir. Environmental Science and Technology, 2014, 37(1) : 60-64. [袁轶君, 毕永红, 朱孔贤等. 三峡水库沉积物中碱性磷酸酶的活 性. 环境科学与技术, 2014, 37(1): 60-64.]

[18] Zhang P, Feng J, Li Z et al. Alkaline phosphatase activity and its kinetics in Lake Gaoyang, Pengxi River during high water level. J Lake Sci, 2015, 27 (4) : 629-636. DOI : 10.18307/2015.0410. [张萍, 冯婧, 李哲等. 三峡澎溪河高阳平 湖高水位时碱性磷酸酶活性及其动力学特征. 湖泊科学, 2015, 27(4) : 629-636.]

[19] Yuan YJ, Bi YH, Hu ZY et al. Phytoplankton communities determine the spatio-temporal heterogeneity of alkaline phosphatase activity: evidence from a tributary of the Three Gorges Reservoir. Scientific Reports, 2017, 7. DOI: 10.1038/ s41598-017-16740-4.

[20] Ministry of Environmental Protection of the People's Republic of China, Editorial Board of Water and Wastewater Monitoring and Analysis Methods eds. Water and wastewater monitoring and analysis methods: 4th edition. Beijing: China Environmental Science Press, 2002. [ 国家环境保护总局《水和废水监测分析方法》编委会. 水和废水监测分析方法: 第 4 版. 北京: 中国环境科学出版社, 2002.]

[21] Zhang ZS, Huang XF eds. Methods of freshwater plankton research. Beijing: Science Press, 1991.[章宗涉, 黄祥飞. 淡 水浮游生物研究方法. 北京: 科学出版社, 1991.]

[22] Hu HJ, Wei YX eds. Chinese freshwater algae-System, classification and ecology. Beijing: Science Press, 2006. [ 胡鸿 钧, 魏印心. 中国淡水藻类——系统, 分类及生态. 北京: 科学出版社, 2006.]

[23] Gage MA, Gorham E. Alkaline phosphatase activity and cellular phosphorus as index of the phosphorus status of phytoplankton in Minnesota Lakes. Freshwater Biology, 1985, 15(2) : 227-233. DOI: 10.1111/j.1365-2427.1985.tb00195.x.

[24] Boon PI. Organic matter degradation and nutrient regeneration in Australian freshwaters.1. Methods for exoenzyme assays in turbid aquatic environments. Archiv fur Hydrobiologie, 1989, 115: 339-359.

[25] Zhang CY, Cheng XY, Wang JJ et al. Spatial-temporal variations of alkaline phosphatase activity in the Kuilei Lake and their influencing factors. Chinese Journal of Applied \& Environmental Biology, 2013, 19(3) : 489-494. DOI: 10.3724/sp. j.1145.2013.00489. [张成艳, 成小英, 王建军等. 傀儡湖水体中碱性磷酸酶活性的时空变化及其影响因素. 应用 与环境生物学报, $2013, \mathbf{1 9}(3): 489-494$. ]

[26] Chrost RJ, Siuda W, Halemejko G. Longterm studies on alkaline phosphatase activity (APA) in a lake with fish-aquaculture in relation to lake eutrophication and phosphorus cycle. Archiv fur Hydrobiologie, 1984, 70(1) : 1-32.

[27] China National Environmental Monitoring Centre. Lake( reservoir) eutrophication evaluation method and classification technical provisions, 2001. [中国环境监测总站. 湖泊(水库) 富营养化评价方法及分级技术规定, 总站生字 [2001] 090 号. 2001.]

[28] Zhou YY, Fu YQ. Phosphatases in natural water: origin, charaeteristies and ecological significance. J Lake Sci, 1999,11 (3) : 274-279. DOI: 10.18307/1999.0313. [ 周易勇, 付永清. 水体磷酸酶: 来源、特征及其生态学意义. 湖泊科学, $1999,11(3): 274-279$. ]

[29] Zhou YY, Li JQ, Chen XD et al. Activity, kinetics and spatial variation of dissolved alkaline phosphatase in Lake Donghu. Environmental Science, 1997, 18(5) : 37-40. DOI: 10.1007/BF02951625. [周易勇, 李建秋, 陈旭东等. 东湖溶解态 磷酸酶的活性、动力学特征及其空间分布. 环境科学, 1997, 18(5) : 37-40.]

[30 ] Zhang TX, Wang XR, Jin XC. Vertical variation of alkaline phosphatase activity and phosphorus forms in the Taihu Lake sediment. Journal of Agriculture and Environment Science, 2007, 26(1): 36-40. [章婷䂀, 王晓蓉, 金相灿. 太湖沉积 物中碱性磷酸酶活力 ( APA) 和磷形态的垂向特征及相关性. 农业环境科学学报, 2007, 26(1) : 36-40.]

[31] Jones JG. Studies of freshwater miro-organims: phosphatase activity in lakes of differing degrees of eutrophication. Journal of Ecology, 1972, 60(3): 777-779.

[32] Alvarez S, Guerrero MC. Enzymatic activities associated with decomposition of particulate organic matter in two shallow 
ponds. Soil Biological and Biochemistry, 2000, 32(13) : 1941-1951. DOI: 10.1016/S0038-0717(00)00170-X.

[33] Feuillade J, Feuillade M, Blanc P. Alkaline phosphatase activity fluctuations and associated factors in a eutrophic lake dominated by Oscillatoria rubescens. Hydrobiologia , 1990, 207(1) : 233-240. DOI: 10.1007/BF00041461.

[34] Taga N, Kobri H. Phaphatase activity in entrophic Tokyo Bay. Marine Biology, 1978, 49(3): 223-229. DOI: 10. 1007/BF00391134.

[35] Ye L, Xu YY, Cai QH. The spatial and temporal distribution of nitrate and phosphate in Xiangxi Bay, Three Gorges Reservoir region during the spring bloom period. Acta Hydrobiologica Sinica, 2006, 30(1) :75-79. [叶麟, 徐耀阳, 蔡庆华. 香溪河库湾春季水华期间硝酸盐、磷酸盐的时空分布. 水生生物学报, 2006, 30(1) : 75-79.]

[36] Zhang L, Bi YH, Hu ZY et al. Phosphorus forms and its distribution character in sediment of Three Gorges Reservoir in Hubei. Environmental Science and Technology, 2011, 34(6): 6-9. [张琳, 毕永红, 胡征宇等. 三峡水库湖北段沉积 物磷形态及其分布特征. 环境科学与技术, $2011, \mathbf{3 4}(6)$ : 6-9.]

[37] Stevens RJ, Parr MP. The significance of alkaline phosphatase activity in Loug Neagh. Freshwater Biology, 2006, 7(4) : 351-355. DOI: 10.1111/j.1365-2427.1977.tb01683.x.

[38] Cao XY, Song CL, Zhou YY. Limitations of using extracellular alkaline phosphatase activities as a general indicator for describing P deficiency of phytoplankton in Chinese shallow lakes. Journal of Applied Phycology, 2010, 22 (1) : 33-41. DOI: $10.1007 / \mathrm{s} 10811-009-9422-0$.

[39] Hong HS, Dai MH, Zheng XC. Measurement of alkaline phosphatase activity in sea water substrates and investigation on the role of alkaline phosphatase in the cycling of phosphorus. Oceanologia et Limnologia Sinica, 1992, 23(4) :415-420. [洪华生, 戴民汉, 郑效成. 海水中碱性磷酸酶活力的测定及其在磷的循环中的作用初探. 海洋与湖沼, 1992,23 (4) : 415-420.]

[ 40] Chróst RJ, Overbeck J. Kinetics of alkaline phosphatase activity and phosphorus availability for phytoplankton and bacterioplankton in lake plusee ( North German Eutrophic Lake). Microbial Ecology, 1987, 13 (3) : 229-248. DOI: 10. 1007/BF02025000. 